\title{
Energy Levels and Thermodynamic Properties of the Internal Rotator
}

\author{
J. O. HALFORD \\ Chemistry Department, University of Michigan, Ann Arbor, Michigan
}

(Received August 31, 1949)

\begin{abstract}
To facilitate the calculation of the thermodynamic properties of internal rotators lying outside the limits of available tabulations, methods of determining the energy levels are studied for the purpose of finding the most expeditious route to accurate results. It proves to be unnecessary in any case to calculate many levels from the wave mechanics. A partition function is set up in terms of the correct ground state and a series of approximate levels derived from the old quantum theory, and this is corrected to constancy by the successive substitution of correct levels, starting with the first excited state. A set of levels obtained in this way which gives the correct partition function always leads to correct values of all the thermodynamic properties.

To obtain accurate energy levels, the continued fractions of Koehler and Dennison are recommended as most convenient.
\end{abstract}

$\mathrm{E}^{\mathrm{x}}$ XTENSIVE tables of the four thermodynamic properties of the hindered internal rotator have been published by Pitzer and Gwinn. ${ }^{1}$ These tables are based upon energy levels obtained from rigorously derived relations for a system of two coaxial rotators, one symmetrical, the other "accidentally" symmetrical, and are recommended as accurate enought for the more general case of a rigid framework with non-interacting symmetrical tops attached at any angles. The energy levels come out as regions within which individual levels may appear, such that for any fixed combination of quantum states of the remaining degrees of freedom, a particular level is determined within each region, and, by numerical summation, a partition function and the derivable thermodynamic properties are determined.

For a given temperature, potential barrier, moment of inertia and symmetry number, there are two limiting values of each property of the internal rotator between which the representative average value must lie. Pitzer and Gwinn ${ }^{1}$ found it unnecessary to develop a valid solution for the average, but limited their calculations to those regions of the variables in which the differences between the limiting values are negligible, and found that they had apparently covered all cases for which the tables were likely to be used.

The recent accurate determination of a low potential barrier and a low internal moment of inertia from spectroscopic data for methyl alcohol by Burkhard and Dennison ${ }^{2}$ places this compound well outside the limits of the Pitzer and Gwinn ${ }^{1}$ tabulations and probably means that there will be other cases as well which are not covered by the tables.

As yet there appears to be no basis upon which any considerable general extension of the tables could be founded, and, even if there were, the extension could hardly be justified when few compounds are expected to require it. In order to deal with the methyl alcohol case, for which the thermodynamic properties are now being calculated, available methods of setting up the

1 K. S. Pitzer and W. D. Gwinn, J. Chem. Phys. 10,428 (1942).
2 D. G. Burkhard and D. M. Dennison, private communication. required energy levels, or regions of energy levels, have been examined, and a scheme had been devised which makes liberal use of rapidly calculated old quantum theory levels at higher energies. In this paper this scheme is developed and illustrated with cases lying within the limits of the tables of Pitzer and Gwinn. ${ }^{1}$

The energy levels used by Pitzer and Gwinn ${ }^{1}$ were obtained from Hill's determinant and an equation representing a theorem discovered by Hill. ${ }^{3}$ The description of the actual calculation is extremely brief and is likely to give the reader the impression that the required levels can be obtained only with extensive laborious calculations. An equivalent solution, developed by Koehler and Dennison ${ }^{4}$ for the simple cosine potential function and the symmetry number 3 , is readily generalized for all symmetry numbers, contains the coupling constant within the determinant, thereby eliminating the need for Hill's theorem, and can be expressed in terms of infinite continued fractions which are easier to solve than the determinant. As compared with Hill's determinant, which includes solutions for more complicated potential functions, the simple continued fractions are limited to the form $V=V_{0}(1$ $-\cos n x) / 2$, but they contain all the information necessary to calculate the available tabulated properties.

An outline of the derivation of the continued fractions, following Koehler and Dennison ${ }^{3}$ except for the introduction of the general symmetry number $n$, is given below. The wave equation for the internal motion is:

$$
d^{2} M / d x^{2}+(R+2 \alpha \cos n x) M=0 .
$$

The solution has the form

$$
M=e^{i \sigma x} \sum_{-\infty}^{+\infty} a_{\tau} e^{i \tau x}
$$

in which the $a_{\tau}$ are Fourier coefficients, and $\sigma=-K C_{1} / C$ where $K$ is the quantum number of the rotation of the whole molecule around the axis of the internal rotation,

${ }^{3}$ Hill, Whittaker, and Watson, Modern Analysis (Cambridge University Press, London, 1935), p. 413.

${ }^{4}$ J. S. Koehler and D. M. Dennison, Phys. Rev. 57, 1006 (1940). 
$C_{1}$ is the moment of inertia of one of the coaxial rotators, and $C$ is the sum of the moments of the two rotating parts. The symmetry number of the symmetrical top appears as $n$ in Eq. (1). By substituting from Eq. (2) and its differentiated forms into Eq. (1) and recognizing that the coefficient of each $e^{i r x}$ must be zero, the valid solutions are obtained as those which conform to the recursion formula

$$
\frac{\alpha}{R-(\sigma+\tau)^{2}} a_{\tau-n}+a_{\tau}+\frac{\alpha}{R-(\sigma+\tau)^{2}} a_{\tau+n}=0 .
$$

Equation (3), in effect, divides the $a_{\tau}$ into $n$ sets, for each of which a determinant of the coefficients can be set equal to zero, or a more convenient equation in continued fractions can be written. Present purposes require only the solution derived from those Eqs. (3) which contain $a_{0}, a_{-n}, a_{n}$, etc. The continued fraction is obtained by setting an arbitrary $a_{r}$, where $\tau$ is large, equal to zero, and expressing each lower $a_{\tau}$ in terms of $a_{\tau \sim n}$ until $a_{-n}$ and $a_{n}$ are obtained in terms of $a_{0}$. The Eq. (3) centered on $a_{0}$ then becomes

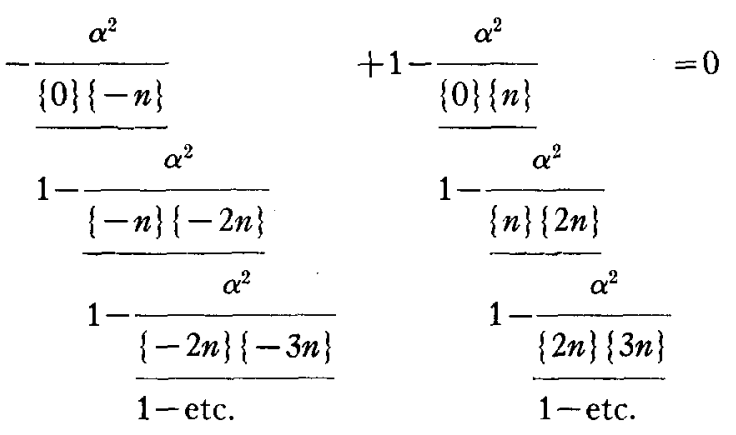

in which $R-(\sigma+n)^{2}$ is represented by $\{n\}$. From the definition of $\alpha$,

$$
\alpha=2 \pi^{2} c C_{1} C_{2} H / h C,
$$

where $H$ is the barrier height in wave numbers and $C_{1} C_{2} / C$ is the reduced internal moment of inertia, combined with the classical partition function of the limiting free rotator,

$$
Q_{f}=\left(8 \pi^{3} C_{1} C_{2} k T / n^{2} C h^{2}\right)^{\frac{1}{2}} ;
$$

it follows that

$$
\alpha=n^{2} Q f^{2} H c h / 4 \pi k T=\left(n^{2} / 4 \pi\right) Q r^{2} V / R T .
$$

In Eq. (7), and subsequently where the fraction $V / R T$ appears, $\mathbf{R}$ is the gas constant and $V$ is the potential barrier in the same energy units. Here $\alpha$ is expressed in terms of the variables tabulated by Pitzer and Gwinn. ${ }^{1}$

For the energy corresponding to a given value of the characteristic number $R$,

$$
W\left(c m^{-1}\right)=h C(R+2 \alpha) / 8 \pi^{2} c C_{1} C_{2}=H(R+2 \alpha) / 4 \alpha
$$

which takes the more convenient form for calculating thermodynamic properties

$$
W / H(=E / V)=R / 4 \alpha+\frac{1}{2},
$$

where the energy appears as a ratio to the barrier height which is independent of the units of energy.

At a fixed value of $\sigma / n$ and a given $Q_{f}$ and $V / \mathrm{R} T$, the $\alpha$ introduced into Eq. (4) is proportional to $n^{2}$, and consequently the derived $R$ will also be proportional to $n^{2}$. Therefore $E / V$ (Eq. (9)) is independent of the symmetry number, and the energy level system at constant $\sigma / n$ will always be the same for a given value of $Q_{f}(V / \mathbf{R} T)^{\frac{1}{3}}$.

The energy levels from Eq. (4) are periodic in $\sigma$, repeating themselves each time this quantity is changed by $n$ units. For a given continuous region of energy levels, all possible numerical values will occur between $\sigma=0$ and $\sigma= \pm n / 2$. To obtain the tabulated results of Pitzer and Gwinn, ${ }^{1}$ two sets of levels, one for $\sigma=0$, the other for $\sigma= \pm n / 2$, are used at each point.

The selection of $-K C_{1} / C$ for $\sigma$ gives the result an apparent asymmetry which is interesting because either moment of inertia could be defined as $C_{1}$. The actual boundary conditions are, more generally, $K C_{1} / C+\sigma=S_{1}$ and $K C_{2} / C-\sigma=S_{2}$ where $S_{1}$ and $S_{2}$ must be integers. If, as in the above definition, either $S_{1}$ or $S_{2}$ is given an arbitrary value, a complete set of energy levels will be obtained, such that, for a given value of $K$, a level of a particular symmetry class always appears at the same energy. The solution, however, may come from any one of the $n$ equations of the type of Eq. (4), according to what integer has been selected for $S_{1}$ or $S_{2}$.

To obtain the ratios $E / V$ (Eq. (9)) which define the energy levels, $\sigma$ is chosen, $\alpha$ is calculated and the $R$ values which satisfy Eq. (4) are found by successive approximation. If the energy regions are numbered off, starting with zero, the condition $\sigma=0$ corresponds to the lower limits of the even numbered regions and the upper limits of the odd-numbered regions. For this condition, Eq. (4) takes the form

$$
\frac{\frac{\alpha^{2}}{\{0\}\{n\}}}{1-\frac{\alpha^{2}}{\frac{\{n\}\{2 n\}}{1-\text { etc. }}}}=0.5 .
$$

When, however, Eq. (10) is used, it develops that only the lower limits of the even numbered regions are directly obtainable, while a singularity is encountered in each region where one of the other solutions is expected to lie. These singularities prove to be valid solutions. Apparently, the derivation of Eq. (4), which involves a succession of divisions, has included an inadvertent division by zero for the case $\sigma=0$. If an appropriate portion of the corresponding determinant of the coefficients is expanded, the determinant is 
TABLE I. Energy levels, internal rotator, $n=3, \alpha^{2}=10$.

\begin{tabular}{|c|c|c|c|c|c|}
\hline \multicolumn{3}{|c|}{$\sigma=0$} & \multicolumn{3}{|c|}{$\sigma= \pm 3 / 2$} \\
\hline$l$ & $R$ & $\Delta E / V$ & $l$ & $R$ & $\Delta E / V$ \\
\hline $\begin{array}{l}0 \\
1 \\
2 \\
3 \\
4 \\
5 \\
6 \\
7,8\end{array}$ & $\begin{array}{r}-1.88346 \\
+8.63274 \\
10.50870 \\
36.14499 \\
36.15251 \\
81.06352 \\
81.06353 \\
144.03528\end{array}$ & $\begin{array}{c}0 \\
0.831381 \\
0.979687 \\
3.006413 \\
3.007008 \\
6.557534 \\
6.557535 \\
11.535888\end{array}$ & $\begin{array}{l}0 \\
1 \\
2 \\
3 \\
4 \\
5 \\
6,7\end{array}$ & $\begin{array}{r}-1.37838 \\
+4.75855 \\
20.43826 \\
20.62572 \\
56.34264 \\
56.34280 \\
110.29631\end{array}$ & $\begin{array}{l}0.039931 \\
0.525097 \\
1.764687 \\
1.779507 \\
4.603177 \\
4.603190 \\
8.868589\end{array}$ \\
\hline
\end{tabular}

reduced to zero by the condition,

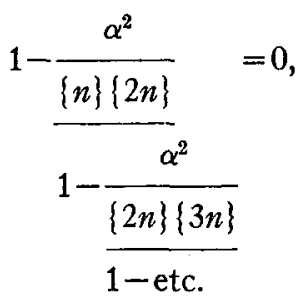

which gives the $R$ values for the upper limits of the oddnumbered regions. Further, for $\sigma=0$, the determinant can be factored into two parts corresponding, respectively, to Eq. (10) and Eq. (11).

At the opposite limits, for $\sigma=-n / 2,\{0\}$ becomes equal to $\{n\},\{-n\}$ to $\{2 n\}$, etc., leading to the equation

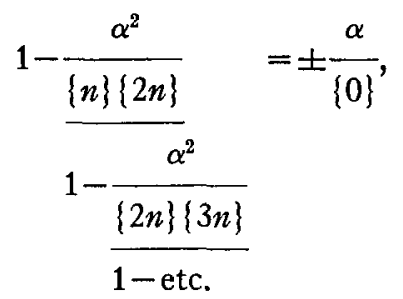

in which the negative roots go with the even-numbered regions, the positive roots with the odd-numbered ones.

To reproduce the tables of Pitzer and Gwinn, ${ }^{1}$ sets of energy levels according to Eqs. (7), (9), (10), (11) and (12) can be obtained for values of $\alpha / n^{2}$ chosen at appropriate intervals, and from each set a series of points running through each table according to Eq. (7) can be obtained, again at chosen intervals. For present purposes, interest is centered upon low values of $\alpha / n^{2}$ for which fewer than three complete regions of levels lie within the potential valley. For higher values of $\alpha / n^{2}$, which cover the major part of the tabulations, it has already been shown that the old quantum theory, with an arbitrary assignment of quantum numbers, will yield accurate results. ${ }^{5}$ These can be obtained most expeditiously with the aid of a subsequently published table. ${ }^{6}$

It is now of interest to inquire, for the region of low $\alpha / n^{2}$, that is, the region of widely spaced levels relative

5 J. O Halford, J. Chem. Phys. 15, 645 (1947).

- J. O. Halford, J. Chem. Phys. 16, 410 (1948), Table II. to the barrier height, how and to what extent the old quantum theory can be used to expedite the calculation without undue loss of accuracy. For this purpose, the limiting free rotator is examined. According to Hill's theorem, the free rotator solutions follow the equation

$$
W=\frac{R h^{2} C}{8 \pi^{2} C_{1} C_{2}} .
$$

If $R$ is the square of an integral multiple of $n$, the solutions correspond to $\sigma=0$, while for $R$ equal to $[n(2 S+1) / 2]^{2}$, where $S$ is an integer, the results are valid for $\sigma= \pm n / 2$. Each level except the lowest is both the upper limit of one region and the lower limit of the next, and the forbidden energies between the eligible regions have disappeared.

Equation (13), for $\sigma=0$, is also the old quantum theory solution for the one-dimensional rotator, and, if the privilege of assigning the intervening half quantum numbers is assumed, the free rotator solution can be said to have been derived from the old quantum theory. Further, since the level system of the hindered rotator rapidly takes on the character of free rotation as the energy increases, there must be some point beyond which the old quantum theory energy levels can be substituted for the more accurate ones without undue loss of accuracy, even when the barrier is appreciable.

The results of a series of old quantum theory graphical integrations are contained in a previously published table $^{6}$ which gives $E / V$ as a function of an index $\rho$ which is defined as:

$$
\rho=r /\left[Q_{f}(V / R T)^{\frac{1}{2}}\right] .
$$

If the hindered rotator is treated like the free rotator, the corresponding assignment of integers and halfintegers to $r$ will again divide the energy space into contiguous regions which should be the same as the correct regions at high energies. At lower energies, the old quantum theory solutions will lie in the forbidden spaces, and each one will represent some kind of an average of the upper limit of one region and the lower limit of the next.

It is proposed to minimize the rather laborious use of Eqs. (10), (11) and (12) in the calculation of thermodynamic properties in the following manner. The old quantum theory limits of the regions are first laid out. Then, starting at the lowest energies, the correct levels are successively substituted until further substitution no longer appreciably changes the partition function or its derivatives. In general, an accurate result will require at least one solution of Eq. (10) and the first pair of solutions of Eq. (12), while the further substitution of accurate levels will depend upon the particular system being evaluated and the desired accuracy of the result.

The proposed method is illustrated below by calculations for the case $\alpha^{2}=10, n=3$, that is, for $1 /\left[Q_{f}(V / \mathrm{R} T)^{\frac{1}{2}}\right]=0.4759$. For any other symmetry number $n^{\prime}$, the same energies would be obtained with 
TABLE II. Energy levels, internal rotator, $1 /\left[Q_{f}(V / R T)^{1}\right]=0.4759$ from old quantum theory graphical integrals.

\begin{tabular}{lccc}
\hline \multicolumn{1}{c}{$l$} & $\sigma=0$ & \multicolumn{2}{c}{$\sigma= \pm 3 / 2$} \\
\hline 0 & $E / V$ & \multicolumn{1}{c}{${ }^{2}$} & \multicolumn{1}{c}{$0 / V$} \\
1,2 & 0 & 0,1 & 0.7413 \\
3,4 & 1.2575 & 2,3 & 2.1209 \\
5,6 & 6.9081 & 4,5 & 4.9545 \\
7,8 & 11.8857 & 6,7 & 9.2198 \\
9,10 & 18.2895 & 8,9 & 14.9144 \\
11,12 & 26.1156 & 10,11 & 22.0490 \\
13,14 & 35.3649 & 12,13 & 30.5470 \\
15,16 & 46.0374 & 14,15 & 40.5244 \\
17,18 & 58.1329 & 16,17 & 51.9085 \\
19,20 & 71.6514 & 18,19 & 64.7158 \\
21,22 & 86.5932 & 20,21 & 78.9460 \\
23,24 & 102.9578 & 22,23 & 94.5993 \\
25,26 & 120.7456 & 24,25 & 111.6757 \\
& & 26,27 & 130.1750 \\
\hline
\end{tabular}

$\alpha^{\prime}=\left(n^{\prime}\right)^{2} \alpha / 9$. For all values of $V / \mathrm{R} T$ below 1.2 , the properties will fall within the limits of the tables of Pitzer and Gwinn. ${ }^{1}$ Table I shows the $R$ values from Eqs. (10), (11) and (12). On the left are the solutions for $\sigma=0$, on the right those for $\sigma= \pm \frac{3}{2}$.

Table II contains the approximations to the same limiting levels given by the old quantum theory.

Calculations are now carried out for $V / \mathrm{R} T=1.0$, $1 / Q_{f}=0.4759$. First, $Q$ is added for the case $\sigma=0$ with all levels except the lowest taken from the old quantum theory, then the sum is corrected by substituting the values for $l=1$ and $l=2$ for the corresponding old quantum theory approximation, the substitution is then made for $l=3$ and 4 , etc., as shown in Table III. For $\sigma=\frac{3}{2}$, the first summation involves entirely old quantum theory levels, referred, however, to the accurate lowest level for $\sigma=0$ as the zero of energies. Substitutions are then made as indicated in the table. Except for the first row, all of the partition functions lead to the same result, $-F / T=1.289,\left(F-F_{f}\right) / T$ $=0.187$. By linear interpolation, from Table II of Pitzer and Gwinn, ${ }^{1}$ the latter value is 0.185 . For this case the free energy has been accurately reproduced with five correct energy levels, although in an independent calculation it would be necessary to increase this number to nine in order to be sure of the accuracy of the result.

In Table IV, the sum $Q^{\prime}=\sum(\Delta E / \mathrm{R} T) e^{-\Delta E / \mathrm{R} T}$ is examined in the same way, for the purpose of calculating $H / T=\mathrm{R} Q^{\prime} / Q$ and $S=(H-F) / T$. The values in the second and subsequent rows, used either with the corresponding $Q$ 's or the limiting $Q$ 's from Table III, all lead to 1.087 for $H / T$, against 1.085 from Pitzer and Gwinn. ${ }^{1}$ The entropy, $S_{f}-S=0.093$, equals the Pitzer and Gwinn value. Again five accurate energy levels suffice.

The heat capacity is now calculated, for which purpose $Q^{\prime \prime}=\sum(\Delta E / \mathrm{R} T)^{2} e^{-\Delta E / \mathrm{R} T}$, as shown in Table $\mathrm{V}$. Again, all of the summations except the first row lead to the same heat capacity, 1.151 at $\sigma=0$, and 1.150
TABLE III. Partition function, internal rotator, $\alpha^{2}=10, n=3$, $1 /\left[Q_{f}(V / R T)^{\frac{1}{3}}\right]=0.4759, V=\mathrm{R} T$, referred to correct zero energy level.

\begin{tabular}{|c|c|c|c|c|c|}
\hline \multicolumn{3}{|c|}{$\begin{array}{c}\qquad \begin{array}{l}\sigma=0 \\
\text { Levels from quantum theory }\end{array}\end{array}$} & \multicolumn{3}{|c|}{$\begin{array}{l}\sigma= \pm 3 / 2 \\
\text { Levels from quantum theory }\end{array}$} \\
\hline New & old & $Q$ & New & Old & $Q$ \\
\hline $\begin{array}{l}0 \\
0,1,2 \\
0-4 \\
\text { all }\end{array}$ & $\begin{array}{l}1 \text { etc. } \\
3 \text { etc. } \\
5 \text { etc. }\end{array}$ & $\begin{array}{l}1.90980 \\
1.91272 \\
1.91265 \\
1.91265\end{array}$ & $\begin{array}{l}0,1 \\
0-3 \\
\text { all }\end{array}$ & $\begin{array}{l}0 \text { etc. } \\
2 \text { etc. } \\
4 \text { etc. }\end{array}$ & $\begin{array}{l}1.71490 \\
1.91337 \\
1.91259 \\
1.91264\end{array}$ \\
\hline
\end{tabular}

TABLE IV. Internal rotator, $\alpha^{2}=10, n=3,1 /\left[Q_{f}(V / \mathrm{R} T)^{1}\right]$ 0.4759, Sum $Q^{\prime}=\Sigma(\Delta E / \mathrm{R} T) e^{-\Delta E / \mathrm{R} T}$.

\begin{tabular}{|c|c|c|c|c|c|}
\hline \multicolumn{3}{|c|}{$\begin{array}{l}\text { Levels from quantum theory } \\
\sigma=0\end{array}$} & \multicolumn{3}{|c|}{$\begin{array}{l}\sigma= \pm 3 / 2 \\
\text { Levels from quantum theory }\end{array}$} \\
\hline New & Old & $Q^{\prime}$ & New & Old & $Q^{\prime}$ \\
\hline $\begin{array}{l}0 \\
0-2 \\
0-4 \\
\text { all }\end{array}$ & $\begin{array}{l}1 \text { etc. } \\
3 \text { etc. } \\
5 \text { etc. }\end{array}$ & $\begin{array}{l}1.04876 \\
1.04624 \\
1.04610 \\
1.04610\end{array}$ & $\begin{array}{l}0,1 \\
0-3 \\
\text { all }\end{array}$ & $\begin{array}{l}0 \text { etc. } \\
2 \text { etc. } \\
4 \text { etc. }\end{array}$ & $\begin{array}{l}1.22606 \\
1.04676 \\
1.04615 \\
1.04615\end{array}$ \\
\hline
\end{tabular}

at $\sigma=\frac{3}{2}$ as against 1.149 from the tables of Pitzer and Gwinn. ${ }^{1}$

For the particular case here illustrated, accurate values of all the thermodynamic properties have been obtained with only five levels derived from the wave mechanics, while the rest are introduced as approximate averages from the old quantum theory. In order to be sure of the result, however, without bringing outside experience into the problem, it would be necessary to increase the number of accurate levels to seven or nine. It appears from these calculations that, whenever the upper limit of one region is close enough to the lower limit of the next, the substitution of an old quantum theory average can be made. Since the required proximity of the two limits is determined by the ratio $E / V$ (i.e., $W / H$ ), the illustration indicates that the substitution can be made whenever this ratio lies above 1.5.

This statement can be tested, with the above set of energy levels, at other values of $V / R T$. If the approximation is accurate at $V / \mathrm{R} T=0.20\left(1 / Q_{f}=0.2128\right)$, it is reasonable to say that it will be satisfactory at all values of $V / \mathrm{R} T$ between 0.20 and 1.00 . The results, with only five accurate levels, three at $\sigma=0$ and two at $\sigma=3 / 2$, are summarized in Table VI. The last row gives the results obtained by interpolation from the tables of Pitzer and Gwinn. ${ }^{1}$ The two correct levels for $l$ equal to 1 and 2 at $\sigma=0$ are not essential to the production of an accurate result at this point. Consequently, the result at $\sigma=0$ can be reproduced exactly as tabulated with only one level correctly evaluated.

To the writer's knowledge, the tabulations of Pitzer and Gwinn have never been independently checked. It may therefore be of some interest that the thermodynamic properties obtained with the energy levels of Table I at several points within the limits of the Pitzer and Gwinn tabulations have always been very close to the tabulated values. A similar set of checks with 
TABLE V. Internal rotator, $\alpha^{2}=10, n=3 ; 1 /\left[Q_{f}(V / \mathrm{R} T)^{1}\right]=0.4759$ $\operatorname{Sum} Q^{\prime \prime}=\Sigma(\Delta E / \mathbf{R} T)^{2} e^{-\Delta E / R T}$.

\begin{tabular}{|c|c|c|c|c|c|}
\hline \multicolumn{3}{|c|}{ 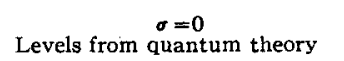 } & \multicolumn{3}{|c|}{$\begin{array}{l}\sigma= \pm 3 / 2 \\
\text { Levels from quantum theory }\end{array}$} \\
\hline New & Old & $Q^{\prime \prime}$ & New & Old & $Q^{\prime \prime}$ \\
\hline $\begin{array}{l}0 \\
0-2 \\
0-4 \\
\text { all }\end{array}$ & $\begin{array}{l}1 \text { etc. } \\
3 \text { etc. } \\
5 \text { etc. }\end{array}$ & $\begin{array}{l}1.68327 \\
1.68078 \\
1.68051 \\
1.68059\end{array}$ & $\begin{array}{l}0,1 \\
0-3 \\
\text { all }\end{array}$ & $\begin{array}{l}0 \text { etc. } \\
2 \text { etc. } \\
4 \text { etc. }\end{array}$ & $\begin{array}{l}1.72034 \\
1.67884 \\
1.67912 \\
1.67914\end{array}$ \\
\hline
\end{tabular}

TABLE VI. Internal rotator at $1 / Q_{f}=0.2128, V / R T=0.2$.

\begin{tabular}{lcccc}
\hline & & & & \\
& $\left(F-F_{f}\right) / T$ & $H / T$ & $S_{f}-S$ & $C$ \\
\hline$\sigma=0$ & 0.055 & 1.043 & 0.005 & 1.002 \\
$\sigma=n / 2$ & 0.055 & 1.043 & 0.005 & 1.003 \\
P \& G & 0.057 & 1.045 & 0.004 & 1.001 \\
\hline
\end{tabular}

energy levels for the case $\alpha^{2}=100,1 /\left[Q_{f}(V / \mathrm{R} T)^{1}\right]$ $=0.26762$, has likewise produced complete agreement. These levels are shown in Table VII.

Tables I and VII contain solutions for fairly high values of $R$, and a few comments upon their derivation from Eqs. (10), (11) and (12) may be in order, although the results of Tables III-VI, inclusive, show that it will not, in general, be necessary to make such evaluations. Since, in general, $R$ must lie between two of the quantities $(\sigma+\tau)^{2}$ and $(\sigma+\tau+n)^{2}$, all of the fractions $\alpha^{2} /[\{\tau\}\{\tau+n\}]$ will be positive except the one for which $R$ lies between the two squares appearing in the denominator. In the numerically evaluated continued fraction, there will therefore be one plus sign, which will appear further down the fraction for the successively higher values of $R$. Beyond this point, the fractions always converge rapidly. Since the continued fractions are equivalent to a determinant of the coefficients of the Eqs. (3), and a solution could be obtained from a limited part of the determinant centered around the point corresponding to the above plus sign, it should be possible to dispense with much of the superstructure of the continued fraction. When the point is reached at which two values of $R$ are indistinguishable (e.g., $l=7,8$ for $\sigma=0$ in Table VII), it is found in this connection that the solution may be obtained by setting a part of the continued fraction equal to zero. For $R$ between $\{\tau\}$ and $\{\tau+n\}$, the following equation gives an accurate enough solution for the
TABLE VII. Energy levels, internal rotator, $n=3, \alpha^{2}=100$.

\begin{tabular}{|c|c|c|c|c|c|}
\hline \multicolumn{3}{|c|}{$\sigma=0$} & \multicolumn{3}{|c|}{$\sigma=3 / 2$} \\
\hline$l$ & $R$ & $\Delta E / V$ & $l$ & $R$ & $\Delta E / V$ \\
\hline 0 & -11.1345 & 0 & 0 & -11.0997 & 0.00087 \\
\hline 1 & 5.5634 & 0.41745 & 1 & 4.8165 & 0.39878 \\
\hline 2 & 16.0374 & 0.67930 & 2 & 20.8324 & 0.79917 \\
\hline 3 & 37.2109 & 1.20864 & 3 & 24.8658 & 0.90001 \\
\hline 4 & 37.8690 & 1.22509 & 4 & 57.1643 & 1.70747 \\
\hline 5 & 81.6375 & 2.31930 & 5 & 57.2140 & 1.70871 \\
\hline 6 & 81.6399 & 2.31936 & 6 & 110.7146 & 3.04623 \\
\hline 7,8 & 144.3330 & 3.88669 & 7 & 110.7147 & 3.04623 \\
\hline
\end{tabular}

higher energy levels.

$$
\begin{aligned}
& 1-\frac{\alpha^{2}}{\frac{\{\tau-2 n\}\{\tau-n\}}{\alpha^{2}}}=0 . \\
& 1-\frac{\alpha^{2}}{\frac{\{\tau-n\}\{\tau\}}{\alpha^{2}}} \frac{\frac{\left.\alpha^{2}\right\}\{\tau+n\}}{1-\text { etc. }}}{\text {. }}
\end{aligned}
$$

From the above examples and the results of an earlier paper, ${ }^{\mathbf{b}}$ the following conclusions can be drawn. If three or more old quantum theory vibrational states lie below the top of the barrier (i.e., if $1 /\left[Q_{f}(V / R T)^{\frac{1}{2}}\right]$ $<0.287)$, the thermodynamic properties can be obtained accurately with the old quantum theory alone. For $1 /\left[Q_{f}(V / \mathbf{R} T)^{\frac{1}{3}}\right]>0.287$, only a few of the now widely spaced lower levels, up to about $E=1.5 \mathrm{~V}$, need to be derived accurately from the wave mechanics. It should be clearly understood that, in the above two regions, the assignment of quantum numbers in the old quantum theory is made in different ways.

For the calculations now in progress for methyl alcohol, which lie outside the limits of present tabulations, it may prove to be necessay, in using old quantum theory levels, to introduce their dependence upon $K$ in a proper way. If $K$, and therefore $\sigma$, is taken for some point between the limits $\sigma=0$ and $\sigma= \pm n / 2$, it is valid to assign the old quantum theory numbers in the free rotator limit on a linear scale. This linear assignment, by analogy to the methods developed here, will then be carried over to the hindered rotator. 